\title{
Significance of salvage autologous stem cell transplantation (ASCT) for patients with multiple myeloma relapsing after up-front ASCT
}

Tsuyoshi Muta1, Toshihiro Miyamoto², Tomoaki Fujisaki ${ }^{3}$, Koichi Osaki ${ }^{4}$, Ryosuke Ogawa ${ }^{1}$, Koji Kato ${ }^{2}$, Kenichi Aoki ${ }^{1}$, Takashi Haro $^{3}$, Tomohiko Kamimura ${ }^{5}$, Kenjiro Kamezaki ${ }^{2}$, Goichi Yoshimoto ${ }^{2}$, Satoshi Yamasaki ${ }^{6}$, Katsuto Takenaka ${ }^{2}$, Yuju Ohno ${ }^{7}$, Hiromi Iwasaki ${ }^{6}$, Testuya Eto ${ }^{8}$, Koji Nagafuji ${ }^{4}$, Koichi Akashi'; Fukuoka Blood and Marrow Transplant Group (FBMTG).

${ }^{1}$ Department of Hematology/Oncology, Japan Community Health Care Organization (JCHO) Kyushu Hospital, Fukuoka ${ }^{2}$ Department of Hematology/Oncology, Kyushu University Hospital, Fukuoka

${ }^{3}$ Department of Internal Medicine, Matsuyama Red-Cross Hospital, Ehime

${ }^{4}$ Division of Hematology and Oncology, department of medicine, Kurume University School of medicine, Fukuoka

${ }^{5}$ Department of Hematology, Hara-Sanshin Hospital, Fukuoka

${ }^{6}$ Department of Hematology, National Hospital Organization Kyushu Medical Center, Fukuoka

${ }^{7}$ Department of Hematology, Kita-kyushu Municipal Medical Center, Fukuoka

${ }^{8}$ Department of Hematology, Hamanomachi Hospital, Fukuoka

We retrospectively analyzed data from 60 patients who had relapsed after up-front autologous stem cell transplantation (ASCT). Thirteen patients received salvage ASCT, and median progression-free survival (PFS) and overall survival (OS) after salvage ASCT were 19.3 and 41.1 months, respectively. Favorable factors for PFS after salvage ASCT were as follows; achieving a partial or better response before salvage ASCT [hazard ratio (HR) = $0.018,95 \%$ confidence interval $(\mathrm{Cl}): 0.005-0.64, P=0.028]$ and consolidation/maintenance therapy after salvage ASCT $(H R=0.083,95 \% \mathrm{Cl}: 0.008-0.87, P=0.038)$. There was no significant factor for OS after salvage ASCT. Patients without salvage ASCT had not been surveyed for PFS after relapse. Including all patients, two unfavorable factors for OS after relapse were as follows; relapsing during or after consolidation/maintenance therapy after initial ASCT and salvage therapy comprising cytotoxic agents. On the other hand, salvage ASCT was favorable for OS after relapse post initial ASCT ( $\mathrm{HR}=0.30,95 \% \mathrm{Cl}: 0.096-0.95, P=0.041)$. Confounding factors and survivor treatment selection bias do not permit definitive conclusion. We hope the current study sheds light on the salvage ASCT, but careful evaluation is needed during the approval of additional new agents. (Journal of Hematopoietic Cell Transplantation $6(2)$ : 98-107, 2017.)

\section{Introduction}

Up-front high-dose melphalan (HD-MEL) followed by autologous hematopoietic stem cell transplantation (ASCT) has been a standard treatment for transplant-eligible patients with multiple myeloma (MM). ${ }^{1}$ Treatment modalities have been intensified using tandem $\mathrm{ASCT}^{2}$ or consolidation therapy. ${ }^{3}$ Maintenance therapy with novel agents have improved the progression-free survival (PFS). ${ }^{4}$ However, subsequent relapse and progression of MM has been inevitable. In Western countries, secondary ASCT with HD-MEL as a salvage therapy (salvage ASCT) for patients who have a relapse after up-font ASCT has been used in clinical practice. The median PFS after salvage ASCT ranged from 5 to 22 months, ${ }^{5-20}$ and the overall response rate ranged from 53.3 to $97.4 \% .^{5,7-15,17-22}$ The median treatment-related mortality rate associated with

Submitted June 29, 2016; Accepted October 11, 2016; Published online, April 14, 2017. (Handling Editor: Chiaki Nakaseko, Chiba University)

Key words: relapsed multiple myeloma, autologous stem cell transplantation, salvage therapy, novel agents

Correspondence: Tsuyoshi Muta, Department of Hematology/Oncology, Japan Community Health Care Organization (JCHO) Kyushu Hospital, 1-8-1 Kishinoura, Yahata Nishi-ku, Kita-Kyushu, Fukuoka 806-8501, Japan. E-mail: muta0621@ gmail.com dx.doi.org/10.7889/hct-16-022 (C) The Japan Society for Hematopoietic Cell Transplantation. 
salvage ASCT was ranged from $0 \%$ to $14.3 \% .^{5,7-20,22}$ So far, the outcomes of salvage ASCT in Japan have not been published. Accordingly, we aimed to conduct a retrospective study to clarify the outcome of ASCT and its significance on overall survival (OS) in patients with relapsed MM.

\section{Patients and Methods}

\section{Patients}

We retrospectively collected the medical records of the patients with MM who needed salvage therapy for progressive or relapsed disease after up-front ASCT in the hospitals belonging to the Fukuoka Blood and Marrow Transplant Group (FBMTG, a collaborative study group in Japan) between June 2001 and December 2014. The eligibility for salvage ASCT had been judged by each physician based on the conditions consisted of adequate renal, hepatobiliary, pulmonary, and cardiac functions; the presence of sufficient CD34+ cells; and the less toxicity during initial ASCT. All patients gave written informed consent for administration of HD-MEL followed by ASCT. For the endpoint of investigation about the outcome of salvage ASCT and its impact on OS comparing to the group who did not receive salvage ASCT, we excluded the patients who received planned tandem ASCT or planned allogeneic stem cell transplantation as well as those who died within 6 months of the initial ASCT. Board approval for the current retrospective study was obtained from the JCHO Kyushu Hospital, the principal investigating institute of the current study, and from each participating institute. The current study was conducted in accordance with the principles of the Declaration of Helsinki.

\section{Diagnostic criteria}

Treatment outcomes were defined as previously described; ${ }^{23}$ complete response (CR), very good PR (VGPR), partial response $(\mathrm{PR})$, stable disease $(\mathrm{SD})$, and progressive disease (PD). Biological relapse was defined as an increase of $25 \%$ from lowest value in any of the following: in the serum levels of M-protein (absolute increase $\geq 0.5 \mathrm{~g} / \mathrm{dL}$ ), in the urine levels of M-protein $(\geq 200 \mathrm{mg} / 24 \mathrm{~h})$, and/or in difference between involved and uninvolved free light chain levels $(\geq 10$ $\mathrm{mg} / \mathrm{dL}) .{ }^{23}$ Symptomatic relapse was identified the occurrence of any myeloma derived end-organ damage, such as lytic bone lesions and/or hypercalcemia. For the OS analysis, the time of death due to any cause was counted as an event, and the final dates of follow-up or those of allogeneic stem cell transplan- tation were defined as censored. Transplant-associated toxicity was evaluated according to the Common Terminology Criteria for Adverse Events version 4.0. Patients alive without any evidence of disease relapse/progression were censored at the last follow-up. Arbitrary, first relapse was defined as the time of symptomatic or biochemical relapse after the initial ASCT; progression-free interval (PFI) was defined as the time from the initial ASCT to the first relapse. PFS after salvage ASCT was also defined as the interval until progression or death from any cause, with censoring.

\section{Statistical methods}

The statistical analysis of the continuous variables included the $\chi^{2}$ and Fisher's exact test as appropriate. Survival was calculated using the Kaplan-Meier method; the log-rank test and/or the Wilcoxon test were used for comparisons, and Cox proportional hazards models were used to estimate hazard ratios (HR) for OS. Each value of $\mathrm{HR}$ and 95\% confidence interval $(95 \% \mathrm{CI})$ were described. All tests were two-sided, and calculations were performed using R-commander(version 1.6-3） based on R （version 2.13.0; R project for Statistical Computing, Vienna, Austria).$^{24}$

\section{Results}

\section{Background of patients receiving salvage ASCT}

We identified 60 patients with MM who had relapsed after initial ASCT and then received second-line therapy. Among them, $13(22 \%)$ patients received salvage ASCT (Table 1). The patients' ages at salvage ASCT ranged from 44 to 71 years (median: 62 years). Figure 1 indicates the time course of each period in a bar graph, mainly to compare PFS after salvage ASCT. Ten patients (cases 1-5, 7, 9, and 11-13) received induction regimens comprising vincristine, doxorubicin and dexamethasone (VAD); of these, 3 patients (cases 5, 9, and 13) received additional bortezomib plus dexamethasone (BD); another 3 (cases 6, 8, and 10) received BD as an induction therapy. Two patients (cases 3 and 5) received chemo-mobilization for salvage ASCT, and the other 11 patients were re-infused with cryopreserved peripheral blood stem cells harvested before the initial ASCT. The median number of infused CD34+ cells was $3.3 \times 10^{6} / \mathrm{kg}$ (range: $2.1-$ $7.4 \times 10^{6} / \mathrm{kg}$ ). The conditioning regimens consisted of HDMEL $\left(200 \mathrm{mg} / \mathrm{m}^{2}\right.$ in all cases except case 6 , which was consisted of $140 \mathrm{mg} / \mathrm{m}^{2}$ combined with bortezomib $1.3 \mathrm{mg} / \mathrm{m}^{2}$ day-4 and day-1). After the initial ASCT, 4 patients (cases 3, 
Table 1. Patient characteristics

\begin{tabular}{|c|c|c|c|}
\hline & $\begin{array}{l}\text { Salvage-ASCT } \\
\quad(n=13)\end{array}$ & $\begin{array}{l}\text { Standard-therapy } \\
\quad(n=47)\end{array}$ & $P$ \\
\hline Age (y) at first ASCT, median (range) & $58(42-67)$ & $61(45-72)$ & 0.8 \\
\hline Male gender & 10 & 30 & 0.4 \\
\hline \multicolumn{4}{|l|}{ Type of M-protein } \\
\hline IgG & 7 & 26 & 0.9 \\
\hline Non-lgG & 6 & 21 & \\
\hline \multicolumn{4}{|l|}{ International staging system at diagnosis } \\
\hline Stage I & 5 & 13 & 0.3 \\
\hline Stage II & 5 & 16 & \\
\hline Stage III & 1 & 15 & \\
\hline Missing & 2 & 3 & \\
\hline \multicolumn{4}{|l|}{ Induction therapy } \\
\hline VAD & 7 & 23 & 0.6 \\
\hline Bortezomib \pm alkylating & 6 & 21 & \\
\hline Lenalidomide \pm alkylating & 0 & 3 & \\
\hline \multicolumn{4}{|l|}{ Methods of PBSCH } \\
\hline $\mathrm{HD}-\mathrm{CY}$ & 11 & 40 & 0.5 \\
\hline HD-VP-16 & 0 & 2 & \\
\hline G-CSF only & 2 & 5 & \\
\hline \multicolumn{4}{|l|}{$\mathrm{HCTC}-\mathrm{Cl}$ at initial ASCT } \\
\hline Score 0-1 & 13 & 40 & 0.1 \\
\hline Score $>2$ & 0 & 7 & \\
\hline \multicolumn{4}{|l|}{ Performance status at initial ASCT } \\
\hline 0,1 & 13 & 44 & 0.4 \\
\hline$>2$ & 0 & 3 & \\
\hline \multicolumn{4}{|l|}{ Conditioning regimens } \\
\hline HD-MEL & 13 & 31 & 0.014 \\
\hline HD-MEL+bortezomib & 0 & 16 & \\
\hline \multicolumn{4}{|l|}{ Response after initial ASCT } \\
\hline $\mathrm{CR}$ & 0 & 11 & 0.2 \\
\hline VGPR & 5 & 17 & \\
\hline PR & 8 & 18 & \\
\hline SD & 0 & 1 & \\
\hline \multicolumn{4}{|c|}{ Consolidation/maintenance after initial ASCT } \\
\hline Yes & 6 & 17 & 0.5 \\
\hline No & 7 & 30 & \\
\hline \multicolumn{4}{|l|}{ Type of relapse } \\
\hline Biological & 9 & 25 & 0.3 \\
\hline Symptomatic & 4 & 22 & \\
\hline \multicolumn{4}{|l|}{ Salvage regimens as second-line } \\
\hline Bortezomib and/or IMIDs & 13 & 38 & 0.09 \\
\hline Cytotoxic agents only & 0 & 9 & \\
\hline \multicolumn{4}{|l|}{ Response after selvage therapy } \\
\hline PR or better & 8 & 27 & 0.8 \\
\hline SD or PD & 5 & 20 & \\
\hline
\end{tabular}

ASCT, autologous stem cell transplantation; $\mathrm{PBSCH}$, peripheral blood stem cell harvest; $\mathrm{HCTC}-\mathrm{Cl}$ Hematopoietic Cell Transplantation-Specific Comorbidity Index; HD, high dose; MEL, melphalan; IMIDs, immunomodulatory drugs; CR, complete response; VGPR, very good partial response; PR, partial response; SD, stable disease; $\mathrm{PD}$, progressive disease. 


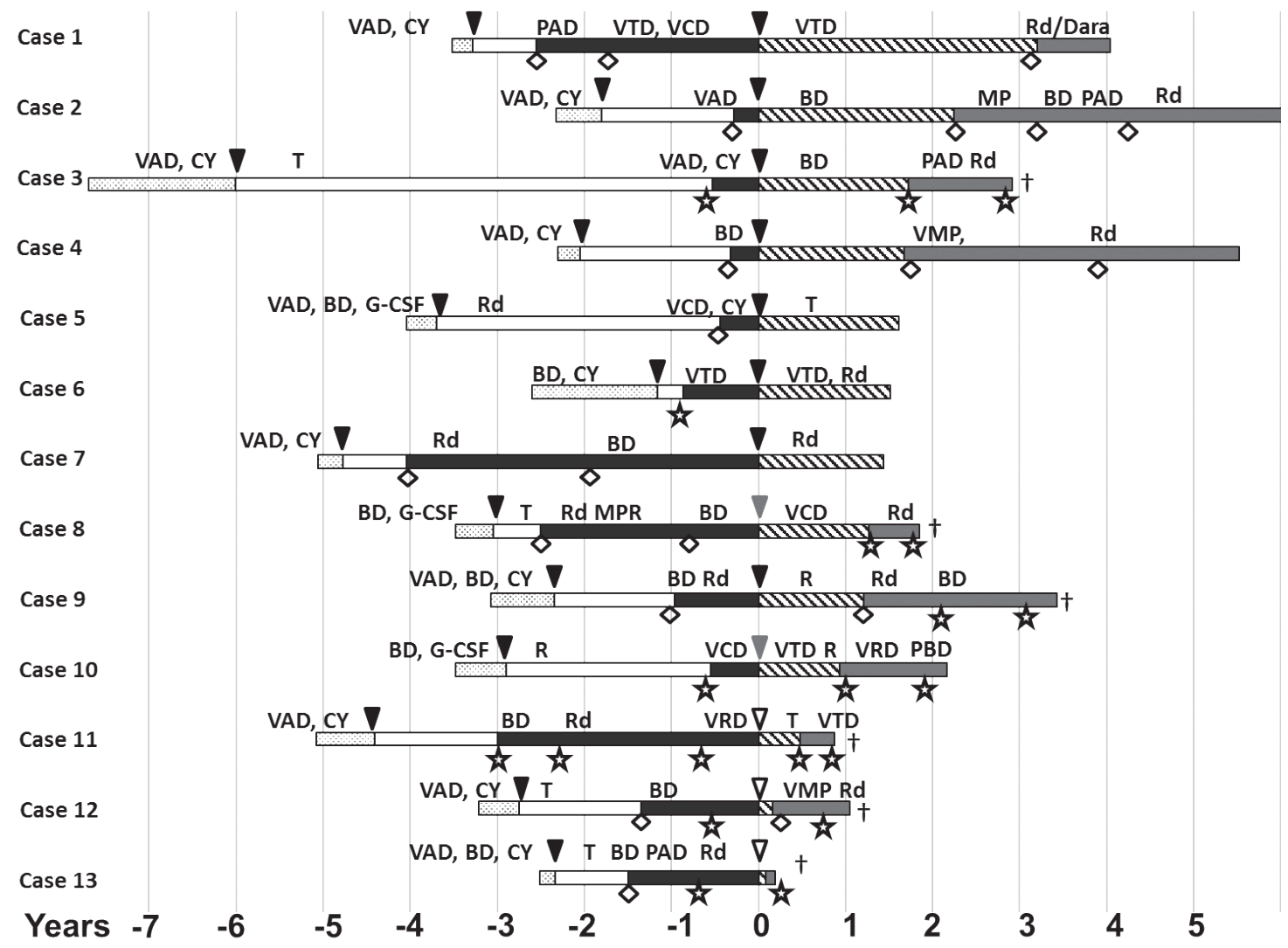

Figure 1. Clinical courses. The bar graph indicates the time sequencing of each patient, depicted as the interval between the initial therapy to the date of final follow-up or death. Each case was aligned at the day of salvage autologous stem cell transplantation (ASCT); cases are arranged in descending order of the duration of progression-free survival (PFS) after salvage ASCT. Abbreviations: mesh bar, time since induction to initial ASCT; white bar, time since initial ASCT to first relapse; black bar, time since first relapse to salvage ASCT; oblique line bar, time since salvage ASCT to subsequent relapse; gray bar, remaining time; plus symbol, date of death; black downward pointing triangle, date of transplantation in at least partial response state; gray downward pointing triangle, day of transplantation at stable disease state; white downward pointing triangle, day of transplantation with progressive disease; rhombus, biochemical relapse; star, symptomatic relapse; VAD, vincristine, adriamycin, and dexamethasone; $\mathrm{CY}$, high-dose cyclophosphamide followed by stem cell harvest; PAD, bortezomib, adriamycin, and dexamethasone; VTD, bortezomib, thalidomide, and dexamethasone; Rd, lenalidomide and dexamethasone; Dara, daratumumab; T, thalidomide; BD, bortezomib and dexamethasone; VMP, bortezomib, melphalan, and predonine; G-CSF, stem cell mobilization by G-CSF; R, lenalidomide; MPR, melphalan, predonine, and lenalidomide; DEXA, dexamethasone; VRD: bortezomib, lenalidomide, and dexamethasone; PVD, panobinostat, bortezomib, and dexamethasone.

8,12 , and 13) received thalidomide and 2 (cases 5 and 10) received lenalidomide as maintenance therapy. Subsequently, 9 patients (cases 1, 2, 4, 5, 7-9, 12, and 13) had biochemical relapse, and 2 (cases 12 and 13) had symptomatic relapse thereafter. In the remaining 4 patients, symptomatic relapses comprised bone diseases.

Consequently, salvage ASCT was incorporated into second-line therapy in 8 patients (cases $2-7,9$, and 10), thirdline in 4 patients (cases 1,8,12, and 13), and forth-line in one patient (case 11).

\section{Outcome of salvage ASCT}

All patients engrafted until median day 11 (range: day 912). After salvage ASCT, three patients (cases 5-7) achieved VGPR, and six (cases 1-4, 8, and 11) achieved PR for an overall response rate of $69 \%$. In addition to febrile neutrope- nia (grade 3) in all patients, several adverse events were reported, including catheter infection (grade 3) in case 10, cytomegalovirus colitis (grade 3 ) in case 11, and pulmonary edema at engraftment (grade 3 ) in case 13. With adequate therapy, all patients recovered from these adverse events. The conditioning regimens for salvage ASCT consisted of HDMEL $\left(100 \mathrm{mg} / \mathrm{m}^{2}\right.$ in case $3 ; 140 \mathrm{mg} / \mathrm{m}^{2}$ in cases 7,11 , and 12 ; and $200 \mathrm{mg} / \mathrm{m}^{2}$ in the other cases) and were combined with bortezomib $\left(1.3 \mathrm{mg} / \mathrm{m}^{2}\right.$ day-4 and day-1) in cases 6,7 , and 10. Cases 6 and 7 received salvage ASCT while in a PR state and achieved VGPR after salvage ASCT; these patients achieved favorable outcomes, with ongoing remission durations of 18.1 months and 17.2 months, respectively, after salvage ASCT. Ten patients received consolidation/maintenance therapy after salvage ASCT; bortezomib-based therapy was administered to six patients (cases $1-3,6,8$, and 10), lenalid- 
omide-based therapy was administered to four patients (cases $6,7,9$, and 10), and thalidomide was administered to two patients (cases 5,11). A total of 10 patients had a relapse, of them, 7 (cases 3, and 8-13) exhibited the CRAB symptoms. Nine of the 10 patients who had a relapse received salvage therapy with novel agents, and 6 patients (cases 3, 8, 9, and 11-13) died due to disease progression. The case 13 had a relapse and progression of myeloma after one month following salvage ASCT and received best supportive care.

\section{Prognosis of salvage ASCT}

In order to evaluate the efficacy of salvage ASCT, univariate comparisons were made within the salvage ASCT group using the log-rank test. During a median follow-up of 63.2 months (range: $30.3-115.9$ months), the median PFI was 16.8 months (rage: 3.6-65.5 months), and the median PFS after salvage ASCT was 19.3 months (range: 1.0-38.4 months). The length of PFI and PFS did not correlate using the Wilcoxon signed-rank test $(P=0.4)$. Eight patients (cases $1-7$ and 9) received salvage ASCT at least PR state, and the median PFS after salvage ASCT for them was 20.7 months (range: 14.4-38.4 months), whereas that for the other patients was 5.7 months (range: 1-15.2 months), showing significant difference (log-rank, $P=0.0004)$. In a multivariate analysis, achieving at least PR before salvage ASCT was a significantly favorable factor associated with PFS after salvage ASCT $\quad(H R=0.018,95 \%$ CI: $0.005-0.64, P=0.028)$, and consolidation/maintenance therapy after salvage ASCT was preferable for a longer PFS after salvage ASCT $(\mathrm{HR}=$ 0.083 , 95\% CI: $0.008-0.87, P=0.038$; Table 2 ). The median OS after salvage ASCT was 41.1 months (range: $2.3-41.1$ months). In a univariate analysis, the response after salvage ASCT (VGPR or PR vs. SD) was significantly associated with a favorable outcome $(\mathrm{HR}=0.14,95 \% \mathrm{CI}: 0.02-0.76, P=$ 0.023; Table 2). The responses before and after salvage ASCT were statistically confounded. In a multivariate, a positive trend in OS was observed in patients with better response after salvage ASCT, not reaching significance (Table 2).

\section{Significance of salvage ASCT on OS after first relapse}

We compared the outcomes of 13 patients (salvage-ASCT group) with those of 47 patients who did not receive salvage ASCT (standard-therapy group; Table 1). Because of the retrospective nature of the study, we could not collect data for the estimation of PFS after salvage therapy in patients without salvage ASCT. Thus, we evaluated OS after the first relapse.
Baseline characteristics before the initial ASCT, such as age, stage, performance status, and co-morbidity, did not significantly differ between the two groups. Sixteen patients in the standard-therapy group received conditioning regimens comprising MEL combined with bortezomib for initial ASCT; none of the patients in the salvage-ASCT group received these regimens $(P=0.014)$. A log-rank analysis found no significant difference in PFI after initial ASCT between the two groups (16.8 months vs. 17.8 months, $P=0.7$ ). Total 23 (38\%) patients received consolidation/maintenance therapy following initial ASCT, and 3 patients in the salvage-ASCT group and 9 patients in the standard-therapy group had a relapse during consolidation/maintenance therapy. Overall, $52(87 \%)$ patients received novel agents for second-line treatment. As a result, the median OS since the first relapse among the salvage-ASCT group tended to be superior compared with the other (52.6 months vs. 33.2 months; log-rank $P=0.064$, Wilcoxon $P=0.039$; Figure 2). Using a Cox model, we examined the factors that had influenced OS since the first relapse (Table 3). Based on multivariate analysis, relapse during or after consolidation/maintenance therapy following initial ASCT was associated with poor prognosis $(\mathrm{HR}=5.42$, 95\% CI 1.85-15.89, $P=0.002)$; furthermore, the salvage therapy, comprising cytotoxic agents only, was associated with worse prognosis $(\mathrm{HR}=15.43,95 \% \mathrm{CI}: 4.82-49.44, P<$ $0.0001)$. In contrast, proceeding to salvage ASCT tended to be associated with a favorable outcome $(\mathrm{HR}=0.30,95 \% \mathrm{CI}$ : 0.096-0.95, $P=0.041$; Table 3).

Longer PFI was found in patients who received consolidation/maintenance therapy after initial ASCT than in patients who did not receive consolidation/maintenance therapy (median 24.2 vs. 14.7 months; log-rank $P=0.027$ ). All 23 patients who relapsed during or after consolidation/maintenance therapy had received novel agents before relapse. However, among 37 patients who did not receive consolidation/ maintenance therapy after initial ASCT, 11 patients (29.7\%) received novel agents before relapse, $19(51.4 \%)$ received novel agents after relapse, and $7(18.9 \%)$ did not receive novel agents $(P<0.001)$.

Five patients who received salvage therapy with cytotoxic agents died within 6 months after relapse, including four patients with treatment-related complications. In contrast, among 38 patients who received salvage therapy with novel agents and no salvage ASCT, five patients died within 6 months, and among 13 patients who received salvage ASCT, no patient died within 1 years after relapse. After removing 


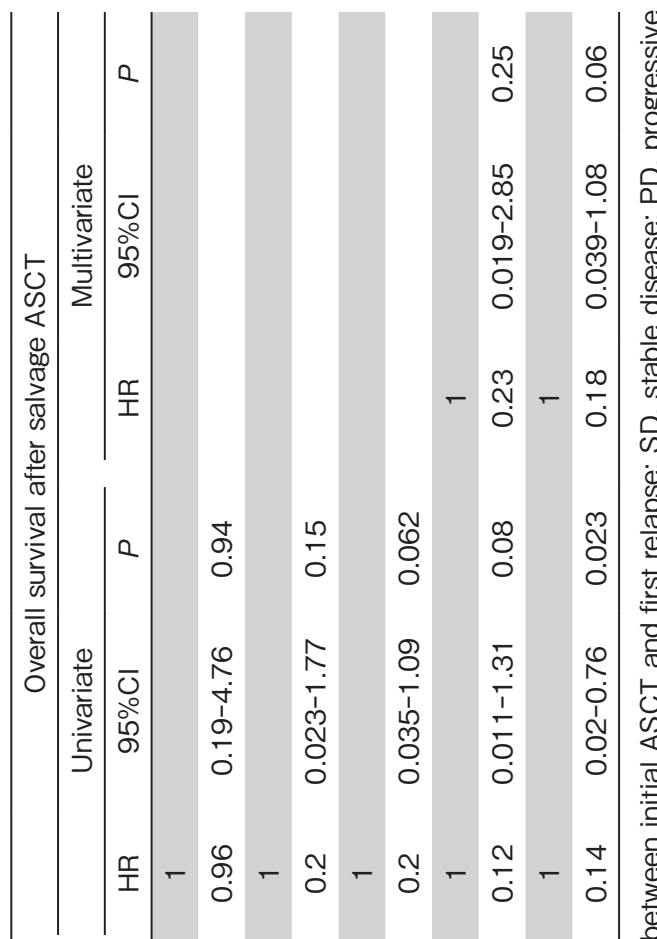

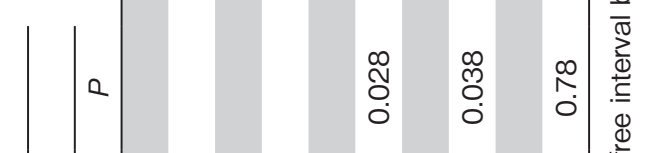

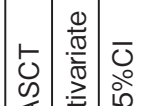

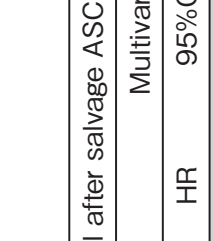

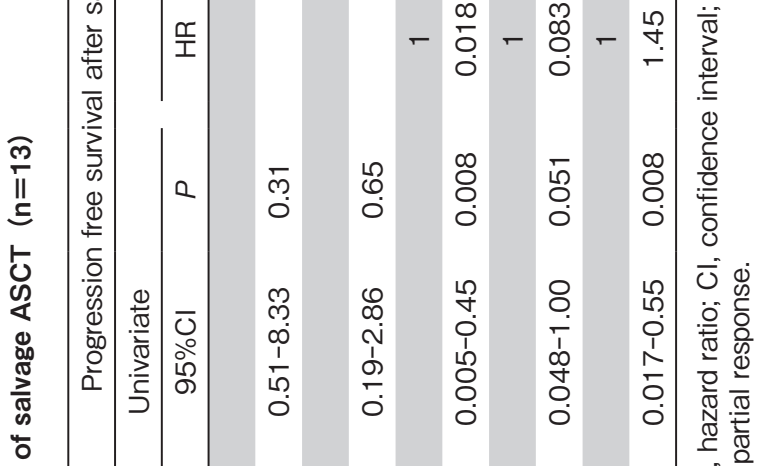

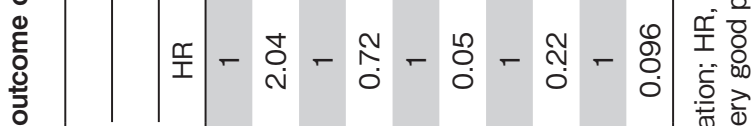

$\stackrel{8}{\rightleftarrows}$

喜

$\frac{8}{8}$

ำ
15

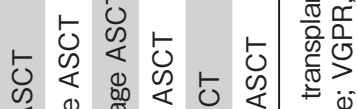

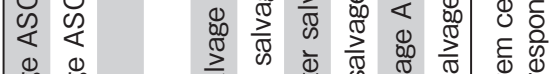

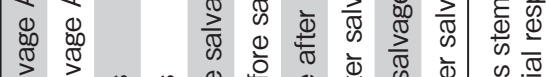

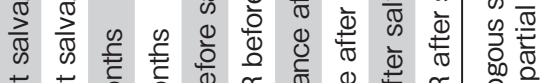
क क

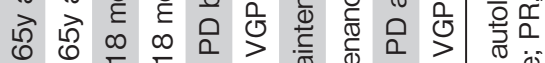

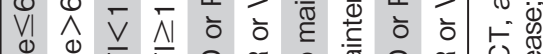

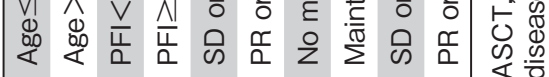

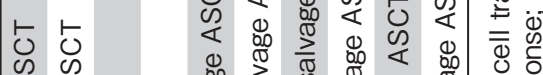

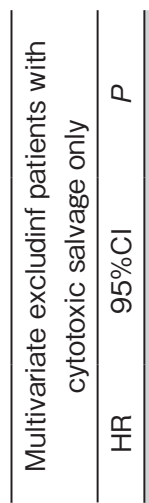

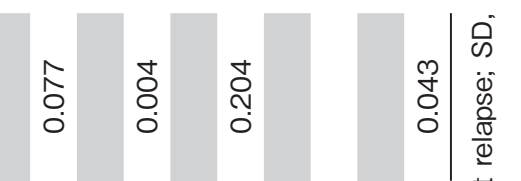

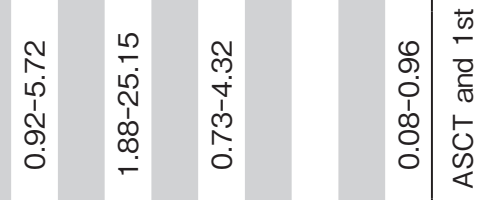

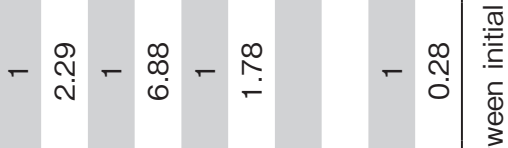

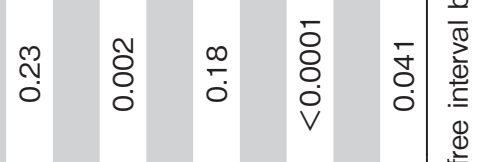

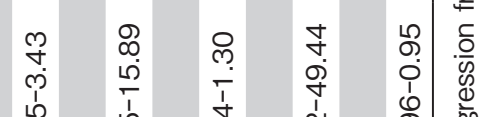

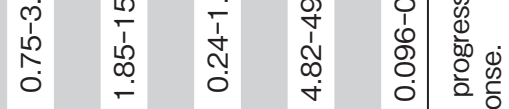

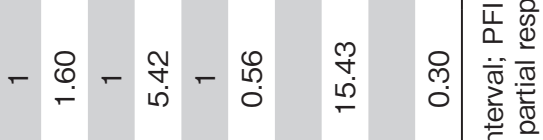

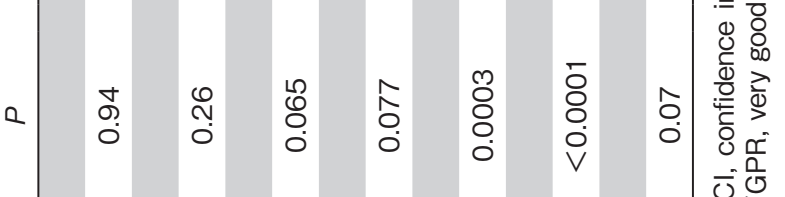

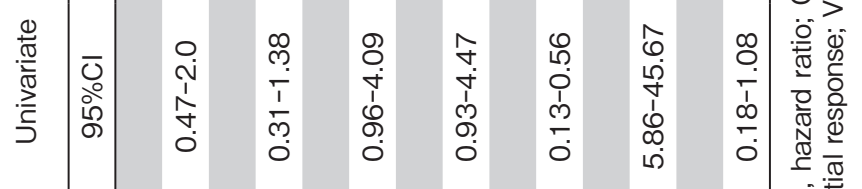

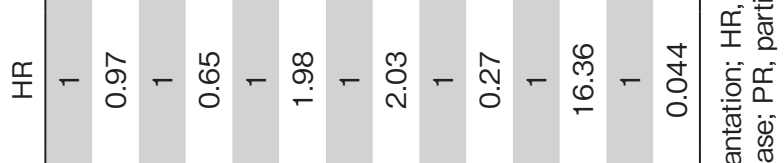

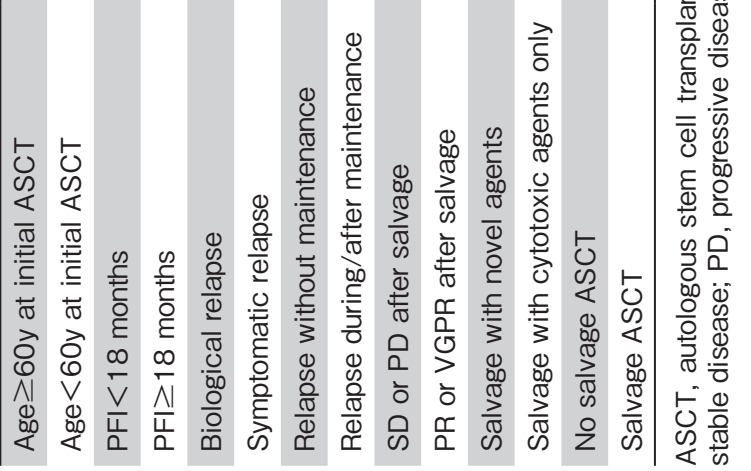


Figure 2. Survival outcomes. Comparison of overall survival since the first relapse after initial autologous stem cell transplantation (ASCT) between the two treatment modalities: salvage ASCT and standard therapy.

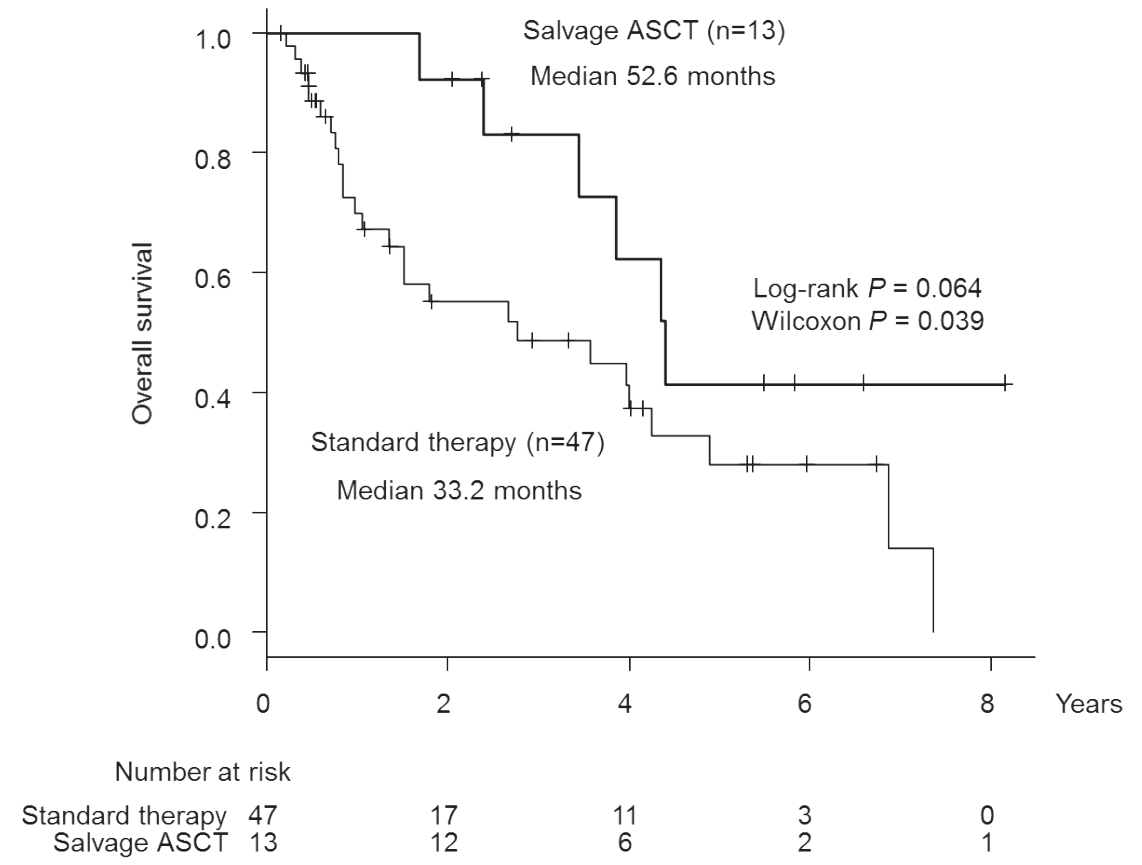

nine patients who received salvage therapy with cytotoxic agents from the analysis, multivariate analysis showed a favorable outcome in patients who received salvage ASCT $(\mathrm{HR}=0.28,95 \%$ CI: 0.08-0.96, $P=0.043$; Table 3). Actually, the patients who received salvage ASCT survived until salvage ASCT, and the time interval should be assessed for survivor treatment selection bias. However, we could not correct the bias enough because of the small sample size in the current analysis.

\section{Discussion}

We demonstrated the pre-transplant disease status was crucial for achieving favorable results after salvage ASCT. Eight patients received salvage ASCT in at least PR state, and the median PFS after salvage ASCT was 20.7 months (range: 14.4-38.4 months). Auner et al. ${ }^{16}$ also reported that a disease status of at least PR before salvage ASCT was identified to be associated with a longer PFS after salvage ASCT. Furthermore, the response after salvage ASCT was closely associated with favorable OS, ${ }^{14,18,19}$ which was also correlated with a favorable outcome in the current study.

The efficacy and tolerability of initial ASCT is critical for employment of salvage ASCT. For the purpose of assessment of initial ASCT, response duration after initial ASCT named PFI in the current study has been evaluated. Regarding PFI between the initial ASCT and first relapse, 12 studies ${ }^{6,10,12-14,16-20,22,25}$ clarified that this longer PFI with the cut- off points of 12 to 36 months and had a significant impact on the favorable outcomes of salvage ASCT. Sellner et al. ${ }^{25}$ successfully demonstrated the risk stratification using International Staging System (ISS) before salvage ASCT and PFI with the cut-off point of 18 months after initial ASCT. The risk groups are categorized into the low-risk group (PFI $>18$ months and ISS stage I), the intermediate-risk group (not applicable to low and high risk), and the high-risk group (PFI $\leq 18$ months and ISS stage II/III), and the median survival time of each group was 58.5 months, 33.9 months and 13.5 months, respectively. ${ }^{25}$ In the current study, 5 patients revealed a PFI of less than 12 months, and 3 of them received consolidation/maintenance modality after salvage ASCT resulting in a PFS of more than 12 months. Accordingly, we could not demonstrate the significance of longer PFI mainly due to the heterogeneity of receiving novel agents.

Concerning about conditioning regimens in salvage ASCT, melphalan plus busulfan and melphalan plus bortezomib showed the response rates of $83 \%$ and $77 \%$, respectively, both of which seem superior to conventional MEL in response rate of $60 \% .{ }^{13}$ In a prospective phase II study, ${ }^{26} 53$ patients with bortezomib-naïve and post-ASCT-relapsed disease had received bortezomib/dexamethasone and bortezomib plus HD-MEL as a conditioning for salvage ASCT. The response rate after salvage ASCT was $96 \%$, and the PFS and OS after re-induction were 21.6 months and 46.6 months, respectively. ${ }^{26}$ In the current study, 2 patients had received HD-MEL combined with bortezomib and resulted in favorable outcome 
after salvage ASCT. An optimum consolidation/consolidation/maintenance therapy after salvage ASCT has not been established. Lemieux et al. revealed that $20 \%$ of the patients received a consolidation therapy after salvage ASCT, and $37 \%$ received a consolidation/maintenance therapy. ${ }^{19}$ A multivariate analysis revealed the significant effects of these treatment modalities on PFS. ${ }^{19}$ In the current study, 8 patients who received consolidation/consolidation/maintenance therapy with novel agents after salvage ASCT showed favorable outcomes on PFS. However, patients who survived relatively longer after salvage ASCT were able to receive maintenance therapy, and we could not correct such survivor treatment selection bias. In the future, optimum conditioning as well as post-transplant therapy for salvage ASCT should be examined.

Another essential point is the need to secure the auto-graft material for salvage ASCT. If more than $4 \times 10^{6} / \mathrm{kg} \mathrm{CD} 34+$ cells were harvested, we conventionally divided them into two transplantation aliquots. A previous study reported a mean CFU-GM recovery rate of $73.8 \% \pm 4.1 \%$ after 18 months of simplified cryopreservation in 5\% dimethyl sulfoxide (DMSO) at $-80{ }^{\circ} \mathrm{C} .{ }^{27}$ Experimentally, safe preservation periods of at least 5 years have been reported. ${ }^{28}$ Actual preservation periods of median 42 months have been reported in the context of salvage ASCT for MM patients. ${ }^{29}$ In the absence of preserved stem cells, re-mobilization with high-dose cyclophosphamide may be possible, as indicated in two patients in the current study. In the literatures, the rate of patients who required re-mobilization for salvage ASCT ranged from 0 to $76 \%$. $^{9,10,14,17-20}$

The superiority of salvage ASCT on OS needs to be demonstrated. Historically, three retrospective studies ${ }^{18,21,22}$ compared OS between the patients who received salvage ASCT and conventional therapy at that time. Prior to the era of novel agents, Cook et al. ${ }^{22}$ reported the superiority of salvage over conventional chemotherapy for OS. In the era of novel agents, Gonsalves et al. ${ }^{18}$ reported a significance of OS after the first relapse between patients who did and did not receive salvage ASCT (57 months vs. 46 months; log-rank $P=0.08$, Wilcoxon $P=0.01$ ), with results that were quite similar to those in the current study. Grövdal $\mathrm{M}$ et al. ${ }^{21}$ retrospectively evaluated OS since the beginning of the second-line treatment and observed superior OS among patients who received salvage ASCT compared with those treated using novel agents alone (median: 4.4 years vs. 3.3 years; $\log$-rank $P=0.013$ ).$^{21}$ According to a unique prospective randomized study of patients who received salvage ASCT or oral cyclophosphamide following bortezomib containing salvage therapy, salvage ASCT was found to be superior in terms of PFS $(\mathrm{HR}=$ $0.36,95 \%$ CI $0.25-0.53, P<0.0001) .{ }^{30}$ The serum levels of beta-2 microglobulin at relapse and the results of in situ hybridization (FISH) were associated with prognosis, ${ }^{30}$ but we couldn't collect the results of FISH in the current study.

The actual ratios of patients who proceeded to salvage ASCT were not high as follows: $26(37 \%)$ of 70 patients, ${ }^{7} 83$ $(21 \%)$ of 404 patients, ${ }^{16}$ and $111(20 \%)$ of 564 patients. $^{21}$ These results may be partly explained by clinical eligibility as well as the hesitation toward receiving high-dose therapy again. We should not ignore the lethal toxicity of high-dose therapy, even if the risk has been reported to be quite low. We have to confirm the willingness of patients to accept an additional risk of mortality to improve survival by undergoing salvage ASCT.

Pomalidomide and panobinostat were released for salvage therapy in Japan in 2015, and carfilzomib has recently become available since September 2016. Among patients with lenalidomide-sensitive relapse after ASCT, the novel combination of carfilzomib, lenalidomide, and dexamethasone resulted in a median PFS of 26.3 months. ${ }^{31}$ There remains a growing concern whether salvage ASCT can prolong survival time following such an effective regimen.

In conclusion, we demonstrated favorable outcome of salvage ASCT, especially in patients who achieved at least PR before salvage ASCT. Among all the patients with relapsing after up-front ASCT, two unfavorable factors for OS after relapse were as follows; relapsing during or after consolidation/maintenance therapy after initial ASCT and salvage therapy comprising cytotoxic agents. On the other hand, salvage ASCT was a favorable factor for OS after relapse. Although confounding factors regarding availability of novel agents and survivor treatment selection bias do not permit definitive conclusions, we hope the current study sheds light on the salvage ASCT. During the approval of several new drugs, it is essential to keep examining whether proceeding to salvage ASCT will yield a survival benefit for each patient.

\section{Acknowledgments}

We appreciate the contributions of all medical and nursing staff at the participating hospitals of FBMTG. 


\section{Conflict of interest disclosure}

We have no conflicts of interest and no funding to declare.

\section{References}

1. Ozaki S, Handa H, Saitoh T, et al. Trends of survival in patients with multiple myeloma in Japan: a multicenter retrospective collaborative study of the Japanese Society of Myeloma. Blood Cancer J. 2015; 5: e349.

2. Sunami K, Shinagawa K, Sawamura M, et al. Phase I/II study of tandem high-dose chemotherapy with autologous peripheral blood stem cell transplantation for advanced multiple myeloma. Int J Hematol. 2009; 90: 635-642.

3. Takashima S, Miyamoto T, Kadowaki M, et al. Combination of bortezomib, thalidomide, and dexamethasone (VTD) as a consolidation therapy after autologous stem cell transplantation for symptomatic multiple myeloma in Japanese patients. Int J Hematol. 2014; 100: 159-164.

4. Attal M, Lauwers-Cances V, Marit G, et al. Lenalidomide maintenance after stem-cell transplantation for multiple myeloma. N Engl J Med. 2012; 366: 1782-1791.

5. Krivanová A, Hájek R, Krejcí M, et al. Second autologous transplantation for multiple myeloma patients relapsing after the first autograft- a pilot study for the evaluation of experimental maintenance therapies. Report of the prospective nonrandomized pilot study of the Czech Myeloma Group. Onkologie. 2004; 27: 275-279.

6. Alvares CL, Davies FE, Horton C, et al. The role of second autografts in the management of myeloma at first relapse. Haematologica. 2006; 91: 141-142.

7. Elice F, Raimondi R, Tosetto A, et al. Prolonged overall survival with second on-demand autologous transplant in multiple myeloma. Am J Hematol. 2006; 81: 426-431.

8. Qazilbash MH, Saliba R, De Lima M, et al. Second autologous or allogeneic transplantation after the failure of first autograft in patients with multiple myeloma. Cancer. 2006; 106: 10841089.

9. Burzynski JA, Toro JJ, Patel RC, et al. Toxicity of a second autologous peripheral blood stem cell transplant in patients with relapsed or recurrent multiple myeloma. Leuk Lymphoma. 2009; 50: 1442-1447.

10. Olin RL, Vogl DT, Porter DL, et al. Second auto-SCT is safe and effective salvage therapy for relapsed multiple myeloma. Bone Marrow Transplant. 2009; 43: 417-422.

11. Krejci M, Adam Z, Buchler T, et al. Salvage treatment with upfront melphalan $100 \mathrm{mg} / \mathrm{m}$ (2) and consolidation with novel drugs for fulminant progression of multiple myeloma. Ann Hematol. 2010; 89: 483-487.

12. Blimark C, Veskovski L, Westin J, et al. Melphalan $100 \mathrm{mg} / \mathrm{m}^{2}$ with stem cell support as first relapse treatment is safe and effective for myeloma patients with long remission after autologous stem cell transplantation. Eur J Haematol. 2011; 87:
$117-122$

13. Fenk R, Liese V, Neubauer F, et al. Predictive factors for successful salvage high-dose therapy in patients with multiple myeloma relapsing after autologous blood stem cell transplantation. Leuk Lymphoma. 2011; 52: 1455-1462.

14. Jimenez-Zepeda VH, Mikhael J, Winter A, et al. Second autologous stem cell transplantation as salvage therapy for multiple myeloma: impact on progression-free and overall survival. Biol Blood Marrow Transplant. 2012; 18: 773-779.

15. Shah N, Ahmed F, Bashir Q, et al. Durable remission with salvage second autotransplants in patients with multiple myeloma. Cancer. 2012; 118: 3549-3555.

16. Auner HW, Szydlo R, Rone A, et al. Salvage autologous stem cell transplantation for multiple myeloma relapsing or progressing after up-front autologous transplantation. Leuk Lymphoma. 2013; 54: 2200-2204.

17. Chow AW, Lee CH, Hiwase DK, To LB, Horvath N. Relapsed multiple myeloma: who benefits from salvage autografts? Intern Med J. 2013; 43: 156-161.

18. Gonsalves WI, Gertz MA, Lacy MQ, et al. Second auto-SCT for treatment of relapsed multiple myeloma. Bone Marrow Transplant. 2013; 48: 568-573.

19. Lemieux E, Hulin C, Caillot D, et al. Autologous stem cell transplantation: an effective salvage therapy in multiple myeloma. Biol Blood Marrow Transplant. 2013; 19: 445-449.

20. Michaelis LC, Saad A, Zhong X, et al. Salvage second hematopoietic cell transplantation in myeloma. Biol Blood Marrow Transplant. 2013; 19: 760-766.

21. Grövdal M, Nahi H, Gahrton G, et al. Autologous stem cell transplantation versus novel drugs or conventional chemotherapy for patients with relapsed multiple myeloma after previous ASCT. Bone Marrow Transplant. 2015; 50: 808-812.

22. Cook G, Liakopoulou E, Pearce R, et al. Factors influencing the outcome of a second autologous stem cell transplant (ASCT) in relapsed multiple myeloma: a study from the British Society of Blood and Marrow Transplantation Registry. Biol Blood Marrow Transplant. 2011; 17: 1638-1645.

23. Rajkumar SV, Harousseau JL, Durie B, et al. Consensus recommendations for the uniform reporting of clinical trials: report of the International Myeloma Workshop Consensus Panel 1. Blood. 2011; 117: 4691-4695.

24. Kanda Y. Investigation of the freely available easy-to-use software 'EZR' for medical statistics. Bone Marrow Transpl. 2013; 48: 452-458.

25. Sellner L, Heiss C, Benner A, et al. Autologous retransplantation for patients with recurrent multiple myeloma: a singlecenter experience with 200 patients. Cancer. 2013; 119: 24382446.

26. Gimsing P, Hjertner $\varnothing$, Abildgaard N, et al. Salvage bortezomib-dexamethasone and high-dose melphalan (HDM) and autologous stem cell support (ASCT) in myeloma patients at first relapse after HDM with ASCT. A phase-2 trial. Bone Marrow Transplantation, 2015; 50: 1306-1311. 
27. Makino S, Harada M, Akashi K, et al. A simplified method for cryopreservation of peripheral blood stem cells at -80 degrees $\mathrm{C}$ without rate-controlled freezing. Bone Marrow Transplant 1991; 8: 329-344.

28. Katayama Y, Yano T, Bessho A, et al. The effects of a simplified method for cryopreservation and thawing procedures on peripheral blood stem cells. Bone Marrow Transplant. 1997; 19: $283-287$.

29. Liseth K, Ersvær E, Abrahamsen JF, et al. Long-term cryopreservation of autologous stem cell grafts: a clinical and experimental study of hematopoietic and immunocompetent cells. Transfusion. 2009; 49: 1709-1719.
30. Cook G, Williams C, Brown JM, et al. High-dose chemotherapy plus autologous stem-cell transplantation as consolidation therapy in patients with relapsed multiple myeloma after previous autologous stem-cell transplantation: a randomised, openlabel, phase 3 trial. Lancet Oncol. 2014; 15: 874-885.

31. Hari PN, Song K, Bensinger W, et al. Efficacy and safety of carfilzomib, lenalidomide, and dexamethasone versus lenalidomdie and dexamethasone in patients with relapsed multiple myeloma after autologous stem cell transplantation: secondary analysis from the phase 3 ASPIRE study (NCT01080391). Biol Blood Marrow Transplant. 2016; 22: S66-S67. 